\title{
Comparison of Two Kinds of Large Displacement Precision Parallel Mechanisms for Micro/nano Positioning Applications
}

\author{
Yuan Yun and Yangmin Li \\ Department of Electromechanical Engineering, Faculty of Science and Technology \\ University of Macau \\ Av. Padre Tomás Pereira, Taipa, Macau, China \\ ya77406@umac.mo,ymli@umac.mo
}

\begin{abstract}
This paper presents kinematic analysis of two kinds of large displacement parallel platforms for micro/nano positioning applications. The kinematics model of the dual parallel mechanism systems is established via the stiffness model of individual wide-range flexure hinge. The displacements of the end platform and the input parameters of prismatic actuators are discussed and the corrected values of input motions are proposed on some checking points in workspace referring to the real parameters of two kinds of In-Plane parallel mechanisms. The FEA models are established by ANSYS software, both the theoretical analysis and FEA simulation results are presented and compared. The investigations of this paper will provide suggestions to improve the structure optimization for a class of parallel mechanism in order to achieve such features as larger workspace and higher motion precision.
\end{abstract}

Index Terms - In-plane parallel mechanism, Flexure hinge, Stiffness matrix, FEA model.

\section{INTRODUCTION}

Parallel manipulators offer the advantages of high stiffness, low inertia, and high speed capability at the expense of smaller workspace, more complex mechanical design, difficult direct kinematics and complicated control algorithms. The errors of parallel manipulators are distributed averagely in the serial chains while the errors of serial manipulator are accumulated, therefore parallel manipulators have been intensively investigated by both industry and academic fields in recent years.

Micro/nano positioning devices are increasingly being made of parallel manipulators due to their characteristics of high precision. Some designers adopt the flexure hinges instead of conventional mechanism joints since the backlash and frictions in the conventional joints affect the performances of high precision parallel mechanisms remarkably. Although the adoption of flexure hinges in the parallel mechanism systems increases the high precision, short stroke actuators with nanometer scale level precision result in a very small workspace. A spatial compliant parallel robot with pseudo-elastic flexure hinges is presented in [1]. The material of the flexure hinges is a kind of shape memory alloy (SMA) which offers elastic strains up to $17 \%$. The robot has $3-\mathrm{DOF}$ and a workspace larger than $200 \times 200 \times 60 \mathrm{~mm}^{3}$. Due to the lack of backlash, friction and stick-slip effects within the mechanism, the repeatability and resolution should be better than $1 \mu \mathrm{m}$ and $0.1 \mu \mathrm{m}$, respectively. A parallel structure for macro-micro systems is proposed in [2]. In this new design, the macro-motion (DC motor) and micro motion (PZT actuator) are connected by a parallel structure, the two motions are coupled under one compliant mechanism framework. This kind of parallel structure eliminates the interface between the macro motion mechanism and the micromotion mechanism. At the same time, a kind of dual parallel mechanism is developed [3], called a 6-PSS parallel mechanism and a 6-SPS one, which is integrated with wide-range flexure hinges as passive joints to ensure the large workspace of the whole system and high precision motion. The experimental prototype platform actuated by piezoelectric motors which can realize a centimeter-scale stroke with positioning precision better than $100 \mathrm{~nm}$ and piezoelectric ceramics actuators with a high resolution about $1 \mathrm{~nm}$ and non-backlash design ensures nanometer scale precision over the cubic centimeter workspace [4], [5]. A XYZ-flexure parallel mechanism (FPM) is proposed with large displacement and decoupled kinematics structure [6]. The large-displacement FPM has a large motion range more than $1 \mathrm{~mm}$. Moreover, the FPM can achieve decoupled $X$-, $Y$ - and $Z$-axes translational motions with small cross-axis error less than $1.9 \%$ and small parasitic rotation less than $1.5 \mathrm{mrad}$. A variant of Stewart-Gogh Platform made of PSS-chains for micro-positioning applications is presented in [7], which gives the stiffness analysis by comparing two platforms in terms of PSS-platform and SPS-platform.

Based on our extensive studies on various kinds of parallel mechanisms [8]- [17], this paper compares the precision analysis of two kinds of In-plane parallel platform for micro/nano positioning applications. The nonlinearity of the wide-range flexure hinges cannot be ignored for a precision positioning system and must be compensated in the kinematics model. So the kinematics model of the dual parallel mechanism system is established via the stiffness model of individual wide-range flexure hinge. Referring to the real parameters of these In-Plane parallel mechanisms, FEA model is established by ANSYS software, both the theoretical analysis and FEA simulation results are presented, which prove that the theoretical model is correct. The constraint orientation workspaces of the two 
mechanisms are plotted and compared. Finally, the errors of the input motions of prismatic actuators between the two kinds of In-plane parallel platforms are discussed on some checking points in workspace.

\section{System Description}

The parallel platform is a dual parallel mechanism combining a 6-PSS parallel mechanism with a 6-SPS one. In the 6-PSS parallel structure, the prismatic actuators provide macro motion with micron level accuracy and cubic centimeter workspace. At the same time, the micro motion is provided by 6-SPS structure which can increase the accuracy of the whole system to the nanometer level. The wide-range flexure hinge is a slender shaft configuration which is adopted as passive joint to ensure the large workspace of the whole system with high precision motion. The schematic of the parallel platform with arbitrary prismatic joint orientation is shown in Fig. 1. A reference frame $o-x y z$ is attached to the fixed platform at the center $o$. And local coordinate system $o^{\prime}-x^{\prime} y^{\prime} z$ ' is attached to the moving platform at the center $o^{\prime}$. The length of each limb is $L_{i}$. Let the vector of the geometry center point of moving platform in the $o$ - $x y z$ coordinate system be $\mathbf{r}_{c} . \Re$ is the transformation matrix from $o^{\prime}-x y^{\prime} z^{\prime}$ coordinate system to $o-x y z . \mathbf{r}_{i L}$ is the vector of point $\mathbf{M}_{i}(\mathrm{i}=1,2, \cdots, 6)$ in the local coordinate system. $\mathbf{r}_{i}$ is the vector of point $\mathrm{M}_{i}(\mathrm{i}=1,2, \cdots, 6)$ in $o$ - $x y z$ coordinate system. $\mathbf{r}_{b i}$ is the vector of point $\mathrm{B}_{i}(\mathrm{i}=1,2, \cdots, 6)$ in $o$-xyz coordinate system.

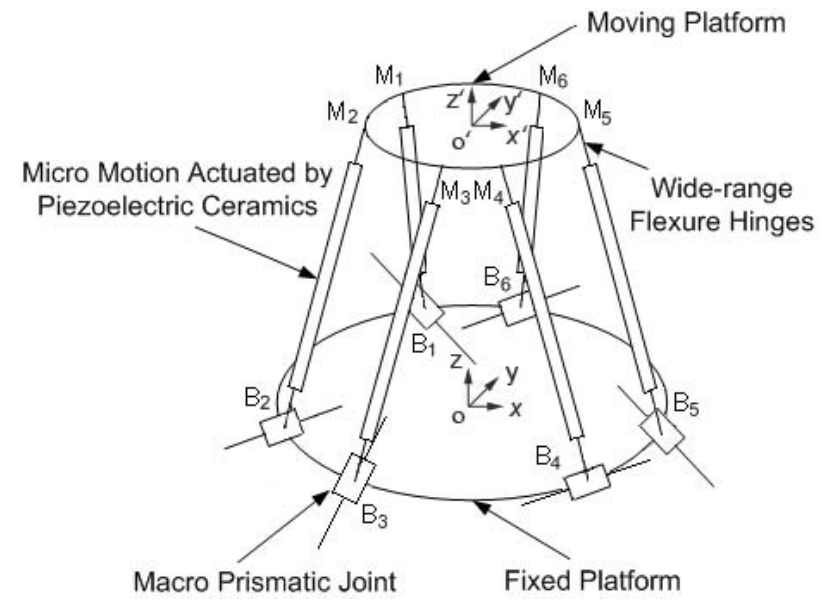

Fig. 1. The parallel platform with arbitrary prismatic joint orientation.

\section{KINEMATICS MODEL}

\section{A. 6-PSS Kinematics Model Based on Stiffness Equation}

Since the large deformation of the wide-range flexure hinges cannot be ignored for a micro/nano positioning applications, the kinematics model is established according to [3]. The widerange flexure hinges and the PZT struts are analyzed as beam elements based on FEM theory. The coordinate systems are established on a single limb of the parallel mechanism as shown in Fig. 2. The single limb is divided into four parts.
Let $\mathbf{T}_{r i}$ be the transformation matrix of the $i^{t h} \operatorname{limb}$ from $o_{i}$ $x_{i} y_{i} z_{i}$ coordinate system to $O-X Y Z . \mathbf{K}_{b i}, \mathbf{K}_{m i}$ and $\mathbf{K}_{s i}$ are the stiffness matrices of the flexure hinge connecting to the fixed platform, the flexure hinge connecting to the moving platform and the PZT strut in $o_{i}-x_{i} y_{i} z_{i}$ coordinate system respectively. $\mathbf{p}_{b i}, \mathbf{p}_{1 i}, \mathbf{p}_{2 i}$ and $\mathbf{p}_{m i}$ are nodal load vectors of these nodes shown in Fig. 6 in $O-X Y Z$ coordinate system. $\mathbf{d}_{b i}, \mathbf{d}_{1 i}, \mathbf{d}_{2 i}$ and $\mathbf{d}_{m i}$ are nodal displacement vector in $O-X Y Z$ coordinate system. Divide the stiffness matrices into $2 \times 2$ sub-matrices:

$$
\mathbf{K}_{b i}=\left[\begin{array}{ll}
\mathbf{K}_{b i_{11}} & \mathbf{K}_{b i_{12}} \\
\mathbf{K}_{b i_{21}} & \mathbf{K}_{b i_{22}}
\end{array}\right]
$$

The stiffness matrix of the whole single limb in $o_{i}-x_{i} y_{i} z_{i}$

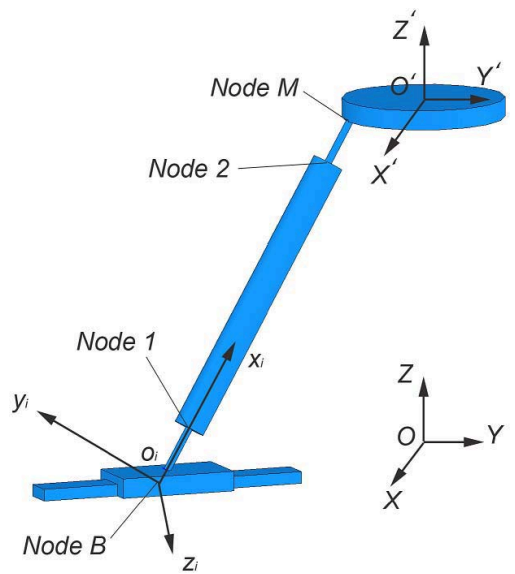

Fig. 2. Single limb of the parallel mechanism.

coordinate system can be achieved by assembling the nodal stiffness matrices:

$$
\mathbf{K}_{i}^{\prime}=\left[\begin{array}{cccc}
\mathbf{K}_{b i_{11}} & \mathbf{K}_{b i_{12}} & \mathbf{0} & \mathbf{0} \\
\mathbf{K}_{b i_{21}} & \mathbf{K}_{b i_{22}}+\mathbf{K}_{s i_{11}} & \mathbf{K}_{s i_{12}} & \mathbf{0} \\
& \mathbf{K}_{s i_{21}} & \mathbf{K}_{s i_{22}}+\mathbf{K}_{m i_{11}} & \mathbf{K}_{m i_{12}} \\
\mathbf{0} & \mathbf{0} & \mathbf{K}_{m i_{21}} & \mathbf{K}_{m i_{22}}
\end{array}\right]
$$

The Lagrange equation of one single limb can be formulated as:

$$
\left[\begin{array}{c}
\mathbf{p}_{b i} \\
\mathbf{p}_{1 i} \\
\mathbf{p}_{2 i} \\
\mathbf{p}_{m i}
\end{array}\right]=\mathbf{T}_{r i}^{T} \cdot \mathbf{K}_{i}^{\prime} \cdot \mathbf{T}_{r i} \cdot\left[\begin{array}{c}
\mathbf{d}_{b i} \\
\mathbf{d}_{1 i} \\
\mathbf{d}_{2 i} \\
\mathbf{d}_{m i}
\end{array}\right]+\mathbf{M} \cdot\left[\begin{array}{c}
\ddot{\mathbf{d}}_{b i} \\
\ddot{\mathbf{d}}_{1 i} \\
\ddot{\mathbf{d}}_{2 i} \\
\ddot{\mathbf{d}}_{m i}
\end{array}\right]
$$

Let

$$
\begin{gathered}
\mathbf{K}_{i}=\mathbf{T}_{r i}^{T} \cdot \mathbf{K}_{i}^{\prime} \cdot \mathbf{T}_{r i} \\
\mathbf{p}_{i}=\left[\begin{array}{c}
\mathbf{p}_{b i} \\
\mathbf{p}_{1 i} \\
\mathbf{p}_{2 i} \\
\mathbf{p}_{m i}
\end{array}\right], \mathbf{d}_{i}=\left[\begin{array}{c}
\mathbf{d}_{b i} \\
\mathbf{d}_{1 i} \\
\mathbf{d}_{2 i} \\
\mathbf{d}_{m i}
\end{array}\right]
\end{gathered}
$$

Eq. (2) can be written as:

$$
\mathbf{p}_{i}=\mathbf{K}_{i} \cdot \mathbf{d}_{i}+\mathbf{M} \cdot \ddot{\mathbf{d}}_{i}
$$

In Eq. (4), the acceleration term can be ignored since the system always works in a low acceleration and uniform motion environment. In addition, let $\mathbf{P}$ be the external load 
vector acting on the moving platform, the force and moment equilibrium equation can be written as:

$$
\mathbf{P}-\sum_{i=1}^{6} \mathbf{p}_{m i}=\mathbf{0}
$$

$\mathbf{d}_{b i}$ is zero vector in terms of solving the deformable error of the moving platform. $\mathbf{d}_{1 i}$ and $\mathbf{d}_{2 i}$ are unknown vectors, and $\mathbf{d}_{m i}(\mathrm{i}=1, \cdots, 6)$ have six unknown elements related to the displacement of the moving platform. $\mathbf{p}_{1 i}$ and $\mathbf{p}_{2 i}$ are zero vectors because of no load acting on node 1 and 2, Eq. (4) can be written as:

$$
\left[\begin{array}{l}
\mathbf{p}_{1 i} \\
\mathbf{p}_{2 i}
\end{array}\right]=\left[\begin{array}{l}
\mathbf{K}_{1 i} \\
\mathbf{K}_{2 i}
\end{array}\right] \cdot\left[\begin{array}{c}
\mathbf{d}_{b i} \\
\mathbf{d}_{1 i} \\
\mathbf{d}_{2 i} \\
\mathbf{d}_{m i}
\end{array}\right]
$$

where

$$
\mathbf{K}_{i}=\left[\begin{array}{c}
\mathbf{K}_{b i} \\
\mathbf{K}_{1 i} \\
\mathbf{K}_{2 i} \\
\mathbf{K}_{m i}
\end{array}\right]
$$

From Eq. (6) for each limb and Eq. (5) that have 78 equations and unknown elements, we can solve the deformation of the moving platform.

When we solve the correcting values of drivers, each $\mathbf{d}_{b i}$ has one unknown element generated by actuator. $\mathbf{d}_{1 i}$ and $\mathbf{d}_{2 i}$ are unknown vectors too, and $\mathbf{d}_{m i}$ can be solved by the displacement of the moving platform. And we can solve the corrected values of actuators from Eq. (6) for each limb and Eq. (5) too.

\section{B. Geometric Nonlinearity of the Wide-range Flexure Hinges}

Since the wide-range flexure hinges are adopted in this parallel mechanism, the system can not be expressed by a linear equation exactly. The stiffness matrix and nodal load is a function of nodal position. Eq. (4) can be written as:

$$
\mathbf{p}_{i}\left(\mathbf{d}_{i}\right)-\mathbf{K}_{i}\left(\mathbf{d}_{i}\right) \cdot \mathbf{d}_{i}-\mathbf{M} \cdot \ddot{\mathbf{d}}_{i}=\phi\left(\mathbf{d}_{i}\right)
$$

where $\phi\left(\mathbf{d}_{i}\right)$ is the imbalance load vector. If $\mathbf{d}_{i}^{*}$ is an accurate solution of Eq. (8), we can obtain:

$$
\mathbf{p}_{i}\left(\mathbf{d}_{i}^{*}\right)-\mathbf{K}_{i}\left(\mathbf{d}_{i}^{*}\right) \cdot \mathbf{d}_{i}^{*}-\mathbf{M} \cdot \ddot{\mathbf{d}}_{i}^{*}=\phi\left(\mathbf{d}_{i}^{*}\right)=\mathbf{0}
$$

Therefore, the Newton-Raphson method is utilized, which uses an iterative process to approach one root of a function. The specific root that the process locates depends on the initial, arbitrarily chosen $\mathbf{d}_{i}$-value.

$$
\mathbf{d}_{i}^{(n+1)}=\mathbf{d}_{i}^{(n)}-\frac{\phi\left(\mathbf{d}_{i}^{(n)}\right)}{\frac{\partial \phi\left(\mathbf{d}_{i}^{(n)}\right)}{\partial \mathbf{d}_{i}^{(n)}}}
$$

The acceleration term can be ignored in static analysis. Assume

$$
\triangle \mathbf{d}_{i}^{(n)}=\frac{\phi\left(\mathbf{d}_{i}^{(n)}\right)}{\frac{\partial \phi\left(\mathbf{d}_{i}^{(n)}\right)}{\partial \mathbf{d}_{i}^{(n)}}}
$$

$$
\mathbf{K}_{\tau}^{(n)}=\frac{\partial \phi\left(\mathbf{d}_{i}^{(n)}\right)}{\partial \mathbf{d}_{i}^{(n)}}=\frac{\partial \mathbf{p}_{i}\left(\mathbf{d}_{i}^{(n)}\right)}{\partial \mathbf{d}_{i}^{(n)}}-\frac{\partial \mathbf{F}_{i}\left(\mathbf{d}_{i}^{(n)}\right)}{\partial \mathbf{d}_{i}^{(n)}}
$$

where $\mathbf{F}_{i}\left(\mathbf{d}_{i}^{(n)}\right)=\mathbf{K}_{i}^{(n)} \cdot \mathbf{d}_{i}^{(n)}$, and $\frac{\partial \mathbf{p}_{i}\left(\mathbf{d}_{i}^{(n)}\right)}{\partial \mathbf{d}_{i}^{(n)}}=0$ in conservative system. Eq. (8) can be written as:

$$
\left\{\begin{array}{l}
\mathbf{K}_{\tau}^{(n)} \cdot \triangle \mathbf{d}_{i}^{(n)}=\phi\left(\mathbf{d}_{i}^{(n)}\right) \\
\mathbf{d}_{i}^{(n+1)}=\mathbf{d}_{i}^{(n)}-\triangle \mathbf{d}_{i}^{(n)}
\end{array}\right.
$$

$\mathbf{K}_{\tau}^{(n)}$ is the tangential stiffness matrix of each limb. It is obviously that the tangential stiffness matrices of each limb have to be updated in order to converge faster to a solution in each sub-step of the iterated algorithm. It is a very huge amount of calculation for a multi-body parallel system. Therefore, a kind of Modified Newton-Rephson method which uses the original tangential stiffness matrix instead of renewal one is adopted to save some time for computations.

\section{6-SPS Kinematics Model}

After the 6-PSS macro motion, the 6-SPS motion is designed to adjust the moving platform in a micro space. The 6SPS can be analyzed by the pseudo-rigid-body model (PRBM). In order to simplify the analysis and design, the PRB model is always proposed for modeling the kinematics input-output behavior of flexure mechanism by expressing a methodology of treating each flexure hinge as a revolute joint with a torsion spring. The model makes analysis of mechanisms with flexure hinges easier and faster.

\section{Simulation Results AND ANALysis}

A FEA model is established with the macro prismatic actuators self-locked as shown in Fig. 3 according to the parameters of the parallel mechanism shown in Table I. The wide-range flexure hinges and struts are meshed with BEAM188 and the material is beryllium bronze and hard aluminum. The moving platform is meshed with SOLID92 and the material is hard aluminum. The external load is $27 \mathrm{~N}$ along $-\mathrm{z}$ axes on the moving platform.

The prismatic actuators directions of the two kinds of

TABLE I

Geometric AND Material Parameters

\begin{tabular}{|l|c|}
\hline Item & Value \\
\hline Radius of moving platform $r$ & $20 \mathrm{~mm}$ \\
\hline Radius of fixed platform $R$ & $90 \mathrm{~mm}$ \\
\hline Angle of short side ratio $\alpha$ & $50^{\circ}$ \\
\hline Radius of flexure hinge $r_{f}$ & $0.9 \mathrm{~mm}$ \\
\hline Length of flexure hinge $l_{f}$ & $12 \mathrm{~mm}$ \\
\hline Radius of strut $r_{s}$ & $4 \mathrm{~mm}$ \\
\hline Length of strut $l_{s}$ & $76 \mathrm{~mm}$ \\
\hline Modulus of elasticity of flexure hinge $E_{f}$ & $130 \mathrm{GPa}$ \\
\hline Modulus of elasticity of strut $E_{s}$ & $70 \mathrm{GPa}$ \\
\hline
\end{tabular}

parallel mechanisms are set to:

$$
\mathbf{a} 1=\left[\begin{array}{lll}
\frac{a(1)}{2} & \frac{\sqrt{3} a(1)}{2} & 0
\end{array}\right]^{T}
$$




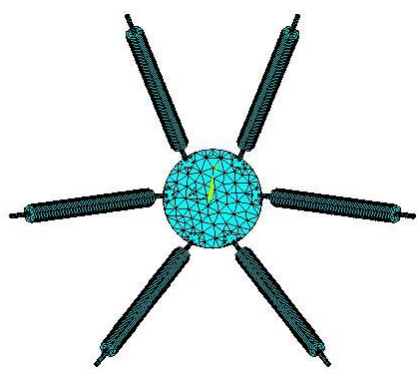

(a)

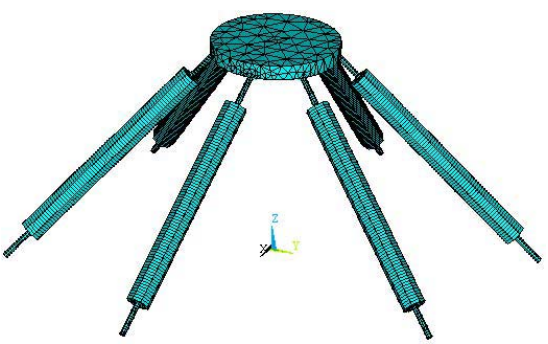

(c)

Fig. 3. FEA model developed in ANSYS.

$$
\begin{gathered}
\mathbf{a} 2=\left[\begin{array}{lll}
-\frac{a(2)}{2} & -\frac{\sqrt{3} a(2)}{2} & 0
\end{array}\right]^{T} \\
\mathbf{a} 3=\left[\begin{array}{lll}
-a(3) & 0 & 0
\end{array}\right]^{T} \\
\mathbf{a} 4=\left[\begin{array}{lll}
a(4) & 0 & 0
\end{array}\right]^{T} \\
\mathbf{a} 5=\left[\begin{array}{lll}
\frac{a(5)}{2} & -\frac{\sqrt{3} a(5)}{2} & 0
\end{array}\right]^{T} \\
\mathbf{a} 6=\left[\begin{array}{lll}
-\frac{a(6)}{2} & \frac{\sqrt{3} a(6)}{2} & 0
\end{array}\right]^{T}
\end{gathered}
$$$$
\mathbf{b} 1=\left[\begin{array}{lll}
-\sin \left(\frac{\alpha}{2}\right) b(1) & \cos \left(\frac{\alpha}{2}\right) b(1) & 0
\end{array}\right]^{T}
$$$$
\mathbf{b} 2=\left[\begin{array}{lll}
-\sin \left(\frac{\alpha}{2}+\beta\right) b(2) & \cos \left(\frac{\alpha}{2}+\beta\right) b(2) & 0
\end{array}\right]^{T}
$$$$
\mathbf{b} 3=\left[\begin{array}{lll}
-\sin \left(\frac{\beta}{2}\right) b(3) & -\cos \left(\frac{\beta}{2}\right) b(3) & 0
\end{array}\right]^{T}
$$$$
\mathbf{b} 4=\left[\begin{array}{lll}
\sin \left(\frac{\beta}{2}\right) b(4) & -\cos \left(\frac{\beta}{2}\right) b(4) & 0
\end{array}\right]^{T}
$$$$
\mathbf{b} 5=\left[\begin{array}{lll}
\sin \left(\frac{\alpha}{2}+\beta\right) b(5) & \cos \left(\frac{\alpha}{2}+\beta\right) b(5) & 0
\end{array}\right]^{T}
$$

$$
\mathbf{b} 6=\left[\begin{array}{lll}
\sin \left(\frac{\alpha}{2}\right) b(6) & \cos \left(\frac{\alpha}{2}\right) b(6) & 0
\end{array}\right]^{T}
$$

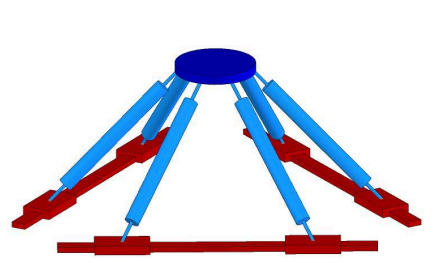

(a)

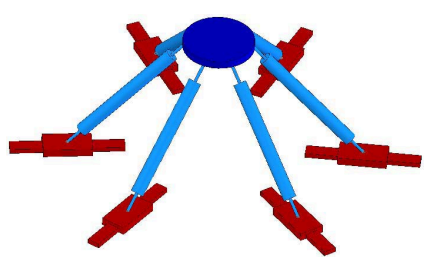

(b)
Fig. 4. The prismatic actuators configurations.

\section{A. Constraint Orientation Workspace}

This kind of 6-PSS parallel mechanism as shown in Fig. 4(a) is a special case with all actuators lying on xy plane and each two of them on one guiding-rail. The errors of moving platform may be decreased through reducing the amount of mounting parts which may introduce more installation errors. The other one is shown in Fig. 4(b) with an In-plane orientations of the actuators along $\overrightarrow{O B_{i}}$. This kind of platforms have superior stiffness values, whereas has very different workspace shape. If the strokes of the prismatic actuators are $-5 \mathrm{~mm}$ $+5 \mathrm{~mm}$, the constraint orientation workspaces are solved by the nonlinear inverse kinematic model as shown in Fig. 5. It is obvious that the structure (b) has a larger workspace than (a) at the same strokes.
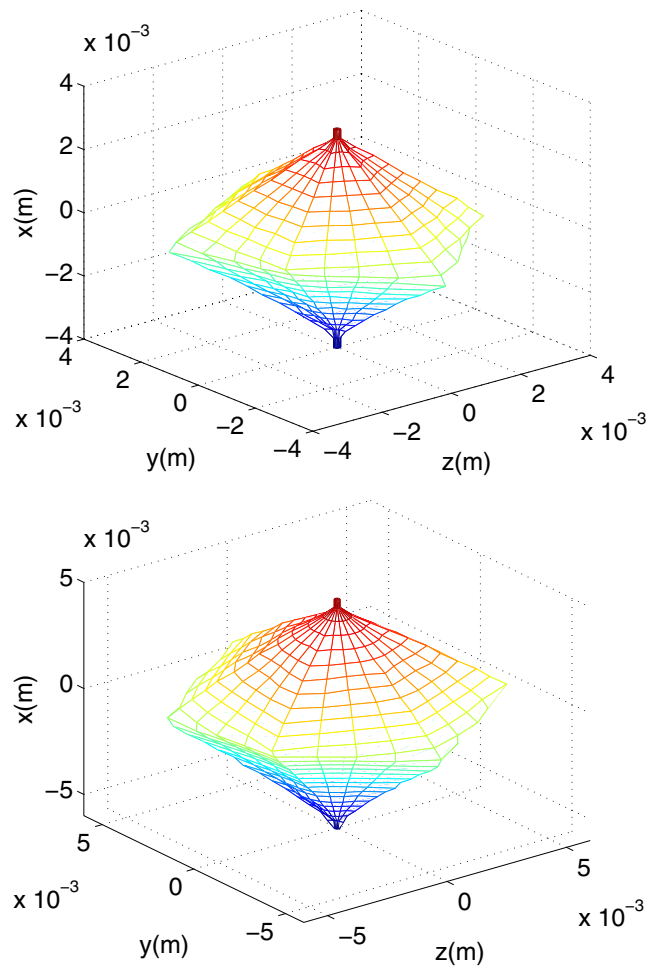

Fig. 5. Constraint orientation workspaces of the macro motions of these two kinds of parallel mechanisms.

\section{B. Simulation Results of Deformation}

According to section II, the kinematics models based on the stiffness matrices are tested by ANSYS. The $z$-axes deformations of the moving platform are plotted in case that the initial position height is $z=71.03 \mathrm{~mm}$ as shown in Fig. 6. One of the ANSYS results is shown in Fig. 7, which has a $27 \mathrm{~N}$ load along $-\mathrm{z}$ axes acted on moving platform.

The figures indicate that two lines are linear and almost the same with the errors under $0.0875 \%$ between the two models. Therefore, the nonlinear results can be tested in ANSYS with the large displacement static analysis. 

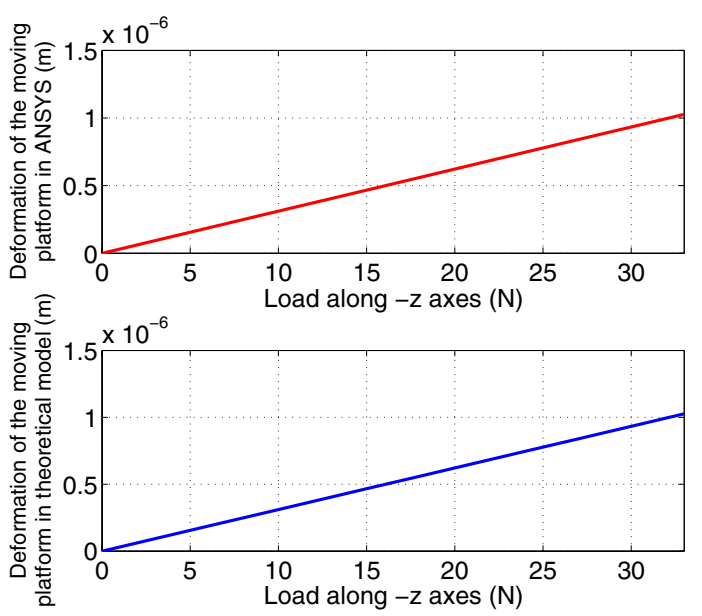

Fig. 6. Simulation results of deformation solved by ANSYS and theoretical model.

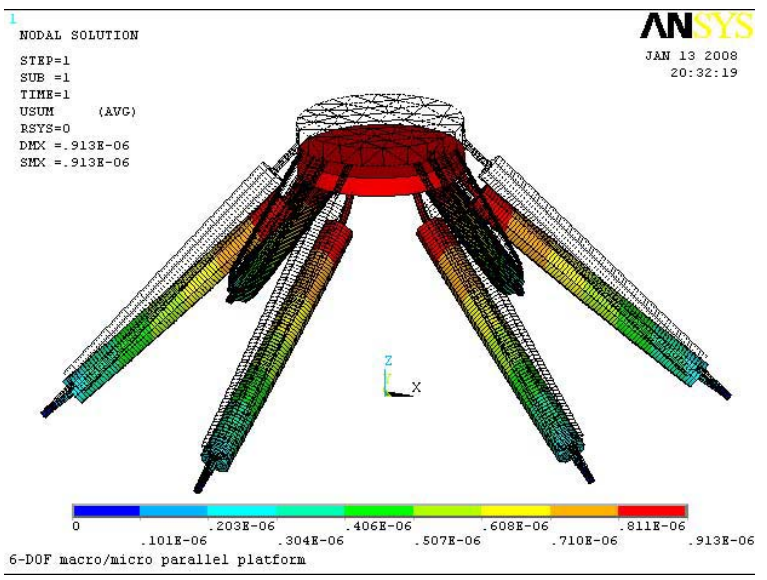

Fig. 7. Simulation result: $27 \mathrm{~N}$ load along $-\mathrm{z}$ axes on moving platform.

\section{Simulation Results of Drive Parameters}

In order to improve the control accuracy, the input parameters of prismatic actuators have to be corrected by the inverse solution of the kinematics model based on the stiffness equations.

In Table II, the input motions of actuators are given by theoretical model and the corrected values of the initial rigid model are listed comparing with the rigid body control. It indicates that the correct values of the checking points are millimeter-scale and gradually increase when the moving platform departures from initial position that can not be ignored in the design of control algorithm for a micro/nano positioning applications. Meanwhile, the correct values of structure (b) are very small compared with (a) and the result showed that the nonlinear effect of structure (a) is getting more serious than that of (b).

The corrected input motions can be tested in ANSYS with the large displacement static analysis. The six chains are constrained via inputting parameters of actuators and fixed all rotation DOF. The wide-range hinges and the moving
TABLE II

Corrected VAlues and InPut Motions of ACtuators

\begin{tabular}{|c|c|c|c|}
\hline \multirow{2}{*}{$\begin{array}{c}\text { Displacements } \\
\left(\mathrm{mm}^{\circ}\right)\end{array}$} & \multicolumn{3}{|c|}{ Corrected Values and Input Motions } \\
\hline & $(\mathrm{mm})$ & (a) & (b) \\
\hline \multirow[t]{2}{*}{ 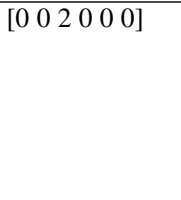 } & $\begin{array}{l}\text { Corrected } \\
\text { values }\end{array}$ & $\begin{array}{ll}(-0.2531 & -0.2531 \\
-0.2531 & -0.2531 \\
0.2531 & -0.2531)\end{array}$ & $\begin{array}{ll}(-0.0714 & -0.0714 \\
-0.0714 & -0.0714 \\
0.0714 & -0.0714)\end{array}$ \\
\hline & $\begin{array}{l}\text { Input } \\
\text { motions }\end{array}$ & 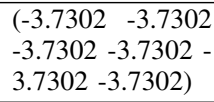 & $\begin{array}{ll}(-2.1513 & -2.1513 \\
-2.1513 & -2.1513- \\
2.1513 & -2.1513)\end{array}$ \\
\hline \multirow[t]{2}{*}{ 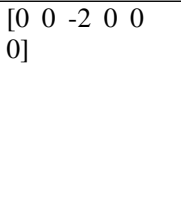 } & $\begin{array}{l}\text { Corrected } \\
\text { values }\end{array}$ & $\begin{array}{ll}(-0.1545 & -0.1545 \\
-0.1545 & -0.1545 \\
0.1545 & -0.1545)\end{array}$ & $\begin{array}{ll}(-0.0588 & -0.0588 \\
-0.0588 & -0.0588 \\
0.0588 & -0.0588)\end{array}$ \\
\hline & $\begin{array}{l}\text { Input } \\
\text { motions }\end{array}$ & $\begin{array}{lr}(2.9764 & 2.9764 \\
2.9764 & 2.9764 \\
2.9764 & 2.9764)\end{array}$ & $\begin{array}{ll}(1.9060 & 1.9060 \\
1.9060 & 1.9060 \\
1.9060 & 1.9060)\end{array}$ \\
\hline \multirow[t]{2}{*}{ 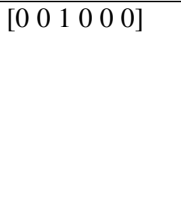 } & $\begin{array}{l}\text { Corrected } \\
\text { values }\end{array}$ & $\begin{array}{ll}-0.0559 & -0.0559 \\
-0.0559 & -0.0559 \\
0.0559 & -0.0559)\end{array}$ & $\begin{array}{ll}(-0.0178 & -0.0178 \\
-0.0178 & -0.0178 \\
0.0178 & -0.0178)\end{array}$ \\
\hline & $\begin{array}{l}\text { Input } \\
\text { motions }\end{array}$ & $\begin{array}{ll}-1.7460 & -1.7460 \\
-1.7460 & -1.7460- \\
1.7460 & -1.7460)\end{array}$ & 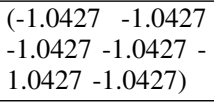 \\
\hline \multirow[t]{2}{*}{ 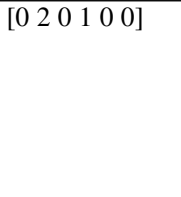 } & $\begin{array}{l}\text { Corrected } \\
\text { values }\end{array}$ & $\begin{array}{ll}(-0.0392 & -0.0335 \\
-0.1460 & -0.2011 \\
0.0523 & -0.0213)\end{array}$ & $\begin{array}{ll}(-0.0022 & -0.0285 \\
-0.0058 & -0.0180 \\
0.0517 & -0.0110)\end{array}$ \\
\hline & $\begin{array}{l}\text { Input } \\
\text { motions }\end{array}$ & 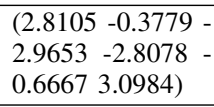 & 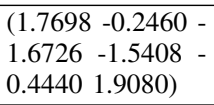 \\
\hline \multirow[t]{2}{*}[\begin{array}{llllll}{0}&{0}&{0}&{3}&{3}&{3}\end{array}]{} & $\begin{array}{l}\text { Corrected } \\
\text { values }\end{array}$ & $\begin{array}{ll}-0.1665 & -0.1612 \\
-0.0165 & -0.1082 \\
0.1542 & -0.0660)\end{array}$ & $\begin{array}{ll}(-0.1019 & -0.0512 \\
-0.0055 & -0.0730 \\
0.0804 & -0.0354)\end{array}$ \\
\hline & $\begin{array}{l}\text { Input } \\
\text { motions }\end{array}$ & $\begin{array}{lr}(-3.1257 & -1.8392 \\
0.3771 & 2.3686 \\
0.8943 & -0.1796)\end{array}$ & $\begin{array}{lr}(-1.9134 & -1.0484 \\
0.2412 & 1.4464 \\
0.5805 & -0.1038)\end{array}$ \\
\hline \multirow[t]{2}{*}{ 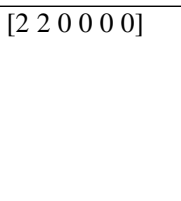 } & $\begin{array}{l}\text { Corrected } \\
\text { values }\end{array}$ & $\begin{array}{ll}-0.0152 & -0.0254 \\
-0.1725 & -0.1633- \\
0.2082 & -0.2063)\end{array}$ & $\begin{array}{ll}(-0.0529 & -0.0297 \\
-0.0042 & -0.0585 \\
0.0403 & -0.0091) \\
\end{array}$ \\
\hline & $\begin{array}{l}\text { Input } \\
\text { motions }\end{array}$ & $\begin{array}{ll}1.7572 & -3.7171 \\
-4.8795 & -0.8703 \\
2.3653 & 3.8314\end{array}$ & $\begin{array}{ll}(0.9955 & -2.3098 \\
-2.8157 & -0.4672 \\
1.6372 & 2.5943)\end{array}$ \\
\hline
\end{tabular}

platform are connected by CONTA175 and TARGE170 elements instead of using $\mathrm{CP}$ command to express rigid constraints as the CP command is invalid in large-deflection effects static analysis. One of the ANSYS results is shown in Fig. 8. The max error ratios of the moving platform are shown in Table III to verify the nonlinear inverse kinematic simulation results.

It can be found that the errors of the checking points between the two kinds of parallel mechanisms are under $10 \%$, when the moving platform departures from initial position, the error ratios are slightly increased which may be produced by the simplified theoretical model. Compared with structure (a), the structure (b) has larger displacement under the same input motions and the $z$-axes motions are with higher accuracy but the $x y$-plane motions and rotated motions are with less accuracy than (a). The configuration of the prismatic actuators is necessary to be optimized for both larger workspace and higher motion precision. 
TABLE III

VERIFICATION OF INVERSE KINEMATIC SIMULATION RESULTS

\begin{tabular}{|c|c|c|}
\hline \multirow{2}{*}{$\begin{array}{c}\text { Displacements } \\
\left(\mathrm{mm}^{\circ}\right)\end{array}$} & \multicolumn{2}{|c|}{ Max Error Ratios Simulated by ANSYS } \\
\hline & (a) & (b) \\
\hline $\left.\begin{array}{llllll}0 & 0 & 2 & 0 & 0 & 0\end{array}\right]$ & $6.29 \%$ & $3.37 \%$ \\
\hline$\left[\begin{array}{llllll}0 & 0 & -2 & 0 & 0 & 0\end{array}\right]$ & $4.84 \%$ & $2.85 \%$ \\
\hline$\left[\begin{array}{llllll}0 & 0 & 1 & 0 & 0 & 0\end{array}\right]$ & $3.28 \%$ & $1.88 \%$ \\
\hline$\left[\begin{array}{llllll}0 & 2 & 0 & 1 & 0 & 0\end{array}\right]$ & $3.88 \%$ & $2.15 \%$ \\
\hline$\left[\begin{array}{llllll}0 & 0 & 0 & 3 & 3 & 3\end{array}\right]$ & $7.07 \%$ & $9.49 \%$ \\
\hline$\left[\begin{array}{llllll}2 & 2 & 0 & 0 & 0 & 0\end{array}\right]$ & $4.01 \%$ & $7.41 \%$ \\
\hline
\end{tabular}

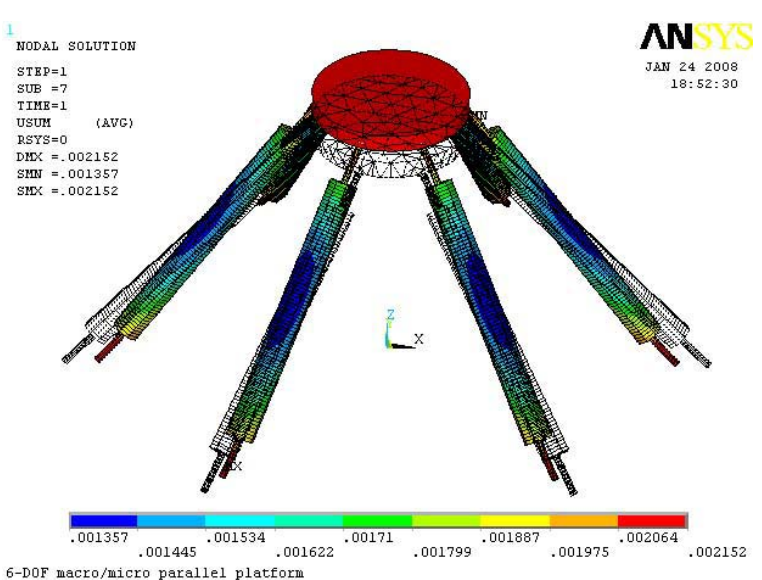

Fig. 8. Simulation result of large displacement static analysis by ANSYS.

\section{CONCLusions}

In this paper, we present precision analysis of two kinds of parallel platform for micro/nano positioning applications. The kinematics model of one class of dual parallel mechanism system is established via the stiffness model of individual wide-range flexure hinges. Referring to the real parameters of an In-Plane parallel mechanism, the constraint orientation workspaces of the macro motions of these two kinds of parallel mechanisms are meshed. FEA model is established in ANSYS, both the theoretical analysis and FEA deformation results are presented in case that the initial position height is $z=71.03 \mathrm{~mm}$, which means that the theoretical model is correct. And the input parameters of prismatic actuators are discussed and the corrected values are proposed on some checking points in workspace. We have found that the structure (b) has a larger workspace than (a) at the same strokes and the correct values of the checking points are millimeter-scale and gradually increase when the moving platform departures from initial position that can extremely influence the control accuracy. Meanwhile, the correct values showed that the nonlinear effect of structure (a) is getting more serious than that of (b). The inverse kinematic simulation results are verified by ANSYS, and the errors of the checking points between the two kinds of parallel mechanisms are under $10 \%$. Compared with structure (a), the structure (b) has larger displacement under the same input motions and the $z$-axes motions are with higher accuracy but the $x y$-plane motions and rotated motions are with less accuracy than (a). The investigations of this paper will provide suggestions to improve the structure and control algorithm optimization for a class of parallel mechanism in order to ensure both larger workspace and higher motion precision. The results will be useful in modifying the structure of the platform with high dynamic properties.

\section{ACKNOWLEDGMENT}

This work was supported by the Research Committee of University of Macau under Grant RG065/0607S/08T/LYM/FST and Macao Science and Technology Development Fund under Grant 069/2005/A.

\section{REFERENCES}

[1] J. Hesselbach, A. Raatz, J. Wrege, and S.Soetebier, "Design and analysis of a macro parallel robot with flexure hinges for micro assembly tasks," Proc. of 35th International Symposium on Robotics, Paris, France, 2004, No.TU14-041fp.

[2] P. R. Ouyang, "Hybrid intelligent machine systems: Design, Modeling and Control," Ph.D. Thesis, University of Saskatchewan, Canada, 2005.

[3] W. Dong, Z. J. Du, and L. N. Sun, "A large workspace macro/micro dual parallel mechanism with wide-range flexure hinges," IEEE International Conference on Mechatronics \& Automation, Niagara Falls, Canada, 2005, Vol. 3, pp. 1592-1597.

[4] W. Dong, L. N. Sun, and Z. J. Du, "Design of a precision compliant parallel positioner driven by dual piezoelectric actuators," Sensors and Actuators A: Physical, Vol. 135, pp. 250-256, 2007.

[5] L. N. Sun, W. Dong, and Z. J. Du, "Loads influence analysis on novel high precision flexure parallel positioner," Chinese Journal of Mechanical Engineering, Vol. 19, No. 1, pp. 37-40, 2006.

[6] X. Tang and I. M. Chen, "A large-displacement 3-DOF flexure parallel mechanism with decoupled kinematics structure," IEEE/RSJ International Conference on Intelligent Robots and Systems, Beijing, China, 2006, pp. 1668-1673.

[7] H. K. Arumugam, R. M. Voyles, and S. Bapat, "Stiffness analysis of a class of parallel mechanisms for micro-positioning applications," IEEE/RSJ International Conference on Intelligent Robots and Systems, Sendai, Japan, 2004, Vol.2, pp. 1826-1831.

[8] Q. Xu and Y. Li, "Design and Analysis of a New Singularity-Free 3PRC Translational Parallel Manipulator," Proceedings of the Institution of Mechanical Engineers, Part C, Journal of Mechanical Engineering Science, Vol. 221, No.5, pp.565-576, 2007.

[9] Y. Li and Q. Xu, "Kinematic Analysis of a 3-PRS Parallel Manipulator," Robotics and Computer-Integrated Manufacturing, Vol. 23, No.4, pp.395408, 2007.

[10] Y. Li and Q. Xu, "Kinematic Analysis and Design of a New 3DOF Translational Parallel Manipulator," Journal of Mechanical Design, Transactions of ASME, Vol. 128, No. 4, pp. 729-737, 2006.

[11] Y. Li and Q. Xu, "A Novel Design and Analysis of a 2-DOF Compliant Parallel Micromanipulator for Nanomanipulation," IEEE Transactions on Automation Science and Engineering, Vol.3, Issue 3, pp.247-254, 2006.

[12] Q. Xu and Y. Li, "A Novel Design of a 3-PRC Translational Compliant Parallel Micromanipulator for Nanomanipulation," Robotica, Vol. 24, No.4, pp. 527-528, 2006.

[13] Q. Xu and Y. Li, "Kinematic Analysis and Optimization of a New Compliant Parallel Micromanipulator," International Journal of Advanced Robotic Systems, Vol.3, No.4, pp.351-358, 2006.

[14] Y. Li and Q. Xu, "Kinematics and Inverse Dynamics Analysis for a General 3-PRS Parallel Mechanism," Robotica, Vol.23, Issue 2, pp. 219229, 2005

[15] Q. Xu and Y. Li, "Mechanical Design of Compliant Parallel Manipulators for Nano Scale Manipulation," IEEE Int. Conf. on Nano/Micro Engineered and Molecular Systems, Zhuhai, China, Jan., 2006, pp. 655-659.

[16] Y. Li and Q. Xu, "Optimal Design of a New Nanopositioner Using Genetic Algorithm," IEEE Int. Conf. on Nano/Micro Engineered and Molecular Systems, Zhuhai, China, Jan., 2006, pp.357-362.

[17] Y. Li, Q. Xu and Y. Liu, "Novel Design and Modeling of a Mobile Parallel Manipulator," IEEE Int. Conf. on Robotics and Automation,Orlando, Florida, USA, May, 2006, pp.1135-1140. 